\title{
The Monge-Ampère equation on almost complex manifolds
}

\section{Szymon Pliś}

Received: 17 August 2012 / Accepted: 22 August 2013 / Published online: 18 October 2013

(C) The Author(s) 2013. This article is published with open access at Springerlink.com

\begin{abstract}
We study the Dirichlet problem for the Monge-Ampère equation on almost complex manifolds. We obtain the existence of the unique smooth solution in strictly pseudoconvex domains.
\end{abstract}

Keywords Monge-Ampère equation $\cdot$ Almost complex manifold $\cdot J$-plurisubharmonic function - Maximal function

Mathematics Subject Classification (2010) $\quad 35 \mathrm{~J} 96 \cdot 35 \mathrm{~B} 65 \cdot 32 \mathrm{Q} 60 \cdot 32 \mathrm{~W} 20$

Let $(M, J)$ be an almost complex manifold of a real dimension $2 n$ (the definitions are given in Sect. 1). Pali proved (in [7]) that, as it is in the case of complex geometry, for plurisubharmonic functions the $(1,1)$ current $i \partial \bar{\partial} u$ is positive. ${ }^{1}$ So for a smooth plurisubharmonic function $u$ we have well defined Monge-Ampère operator $(i \partial \bar{\partial} u)^{n} \geq 0$ and we can study the complex Monge-Ampère equation

$$
(i \partial \bar{\partial} u)^{n}=f d V
$$

where $f \geq 0$ and $d V$ is a (smooth) volume form.

Let $\Omega \Subset M$ be a strictly pseudoconvex domain of class $\mathcal{C}^{\infty}$. In this article we study the following Dirichlet problem for the Monge-Ampère equation:

$$
\left\{\begin{array}{l}
u \in \mathcal{P} \mathcal{S H}(\Omega) \cap \mathcal{C}^{\infty}(\bar{\Omega}) \\
(i \partial \bar{\partial} u)^{n}=d V \text { in } \Omega \\
u=\varphi \text { on } \partial \Omega
\end{array}\right.
$$

where $\varphi \in \mathcal{C}^{\infty}(\bar{\Omega})$. The main theorem is the following:

1 But it can be not closed!.

Partially supported by the project N N201 268335 of the Polish Ministry of Science and Higher Education.

S. Pliś $(\bowtie)$

Institute of Mathematics, Cracow University of Technology,

Warszawska 24, 31-155 Kraków, Poland

e-mail: splis@pk.edu.pl 
Theorem 1 There is a unique smooth plurisubharmonic solution u of the problem (2).

In [3] the theorem above was proved for $\Omega \subset \mathbb{C}^{n}$ with $J_{\text {st }}$. Note that even in the integrable case it is not enough to assume that $\partial \Omega$ is strictly pseudoconvex. ${ }^{2}$ Indeed, if $\Omega$ is the blow-up of a strictly pseudoconvex domain in $\mathbb{C}^{n}$ in one point, then $\partial \Omega$ is strictly pseudoconvex. But if $u \in \mathcal{P} \mathcal{S H}(\Omega) \cap \mathcal{C}^{\infty}(\bar{\Omega})$, then the form $(i \partial \bar{\partial} u)^{n}$ is not a volume form.

In case of $J$ not integrable McDuff constructed a domain $\Omega$ with a non connected strictly pseudoconvex boundary (see [6]). ${ }^{3}$ One can prove the theorem above (in almost the same way) for $\Omega$ not necessary strictly pseudoconvex but $\partial \Omega$ strictly pseudoconvex and $d V \leq(i \partial \bar{\partial} \varphi)^{n}$. It is however not clear for the author, whether there is an example of such $\varphi$ in McDuff's example (or in any other not strictly pseudoconvex domain with a strictly pseudoconvex boundary).

In the last section we explain how Theorem 1 gives the theorem of Harvey and Lawson about existing a continuous solution of the Dirichlet Problem for maximal functions. We even improve their result by proving that the solution is Lipschitz (if the boundary condition is regular enough).

\section{Notion}

We say that $(M, J)$ is an almost complex manifold if $M$ is a manifold and $J$ is an $\left(\mathcal{C}^{\infty}\right.$ smooth) endomorphism of the tangent bundle $T M$, such that $J^{2}=-\mathrm{id}$. The real dimension of $M$ is even in that case.

We have then a direct sum decomposition $T_{\mathbb{C}} M=T^{1,0} M \oplus T^{0,1} M$, where $T_{\mathbb{C}} M$ is a complexification of $T M$,

$$
T^{1,0} M=\{X-i J X: X \in T M\}
$$

and

$$
T^{0,1} M=\{X+i J X: X \in T M\}\left(=\left\{\zeta \in T_{\mathbb{C}} M: \bar{\zeta} \in T^{1,0} M\right\}\right) .
$$

Let $\mathcal{A}^{k}$ be the set of $k$-forms, i.e. the set of sections of $\bigwedge^{k}\left(T_{\mathbb{C}} M\right)^{\star}$ and let $\mathcal{A}^{p, q}$ be the set of $(p, q)$-forms, i.e. the set of sections of $\bigwedge^{p}\left(T^{1,0} M\right)^{\star} \otimes_{(\mathbb{C})} \bigwedge^{q}\left(T^{0,1} M\right)^{\star}$. Then we have a direct sum decomposition $\mathcal{A}^{k}=\bigoplus_{p+q=k} \mathcal{A}^{p, q}$. We denote the projections $\mathcal{A}^{k} \rightarrow \mathcal{A}^{p, q}$ by $\Pi^{p, q}$.

If $d: \mathcal{A}^{k} \rightarrow \mathcal{A}^{k+1}$ is (the $\mathbb{C}$-linear extension of) the exterior differential, then we define $\partial: \mathcal{A}^{p, q} \rightarrow \mathcal{A}^{p+1, q}$ as $\Pi^{p+1, q} \circ d$ and $\bar{\partial}: \mathcal{A}^{p, q} \rightarrow \mathcal{A}^{p, q+1}$ as $\Pi^{p, q+1} \circ d$.

We say that an almost complex structure $J$ is integrable, if any of the following (equivalent) conditions is satisfied:

(i) $d=\partial+\bar{\partial}$;

(ii) $\bar{\partial}^{2}=0$;

(iii) $[\zeta, \xi] \in T^{0,1} M$ for vector fields $\zeta, \xi \in T^{0,1} M$.

By the Newlander-Nirenberg Theorem $J$ is integrable if and only if it is induced by a complex structure.

\footnotetext{
2 We say that $\partial \Omega$ is strictly pseudoconvex if it is locally equal to $\{\rho=0\}$, for some smooth function $\rho$ such that $i \partial \bar{\partial} \rho>0,\{\rho<0\} \subset \Omega$ and $\nabla \rho \neq 0$.

${ }^{3}$ If $\Omega$ is strictly pseudoconvex, then $\partial \Omega$ is connected (see [2]).
} 
In the paper $\zeta_{1}, \ldots, \zeta_{n}$ is always a (local) frame of $T^{1,0}$. Let us put for a smooth function $u$

$$
u_{p}=\zeta_{p} u, \quad u_{p \bar{q}}=\zeta_{p} \bar{\zeta}_{q} u=u_{\bar{q} p}+\left[\zeta_{p}, \overline{\zeta_{q}}\right] u, \text { etc. }
$$

and

$$
A_{p \bar{q}}=A_{p \bar{q}}(u)=u_{p \bar{q}}-\left[\zeta_{p}, \bar{\zeta}_{q}\right]^{0,1} u
$$

where for any $X \in T_{\mathbb{C}} M$ a vector $X^{0,1} \in T^{0,1} M$ is such that $X^{1,0}:=X-X^{0,1} \in T^{1,0} M$. Then for a smooth function $u$ we have (see [7]):

$$
i \partial \bar{\partial} u=i \sum A_{p \bar{q}} \zeta_{p}^{\star} \wedge \bar{\zeta}_{q}^{\star}
$$

where $\zeta_{1}^{\star}, \ldots, \zeta_{n}^{\star}, \bar{\zeta}_{1}^{\star}, \ldots, \bar{\zeta}_{n}^{\star}$ is a base of $\left(T_{\mathbb{C}} M\right)^{\star}$ dual to the base $\zeta_{1}, \ldots, \zeta_{n}, \bar{\zeta}_{1}, \ldots, \bar{\zeta}_{n}$ of $T_{\mathbb{C}} M$.

Let $\mathbb{D}=\{z \in \mathbb{C}:|z|<1\}$. We say that a (smooth) function $\lambda: \mathbb{D} \rightarrow M$ is $J$-holomorphic or simpler holomorphic if $\lambda^{\prime}\left(\frac{\partial}{\partial \bar{z}}\right) \in T^{0,1} M$. The following proposition from [5], where it is stated for $C^{k^{\prime}, \alpha}$ class of $J$, shows that there exists plenty of such disks:

Proposition 1.1 Let $0 \in M \subset \mathbb{R}^{2 n}, k, k^{\prime} \geq 1$. For $v_{0}, v_{1}, \ldots, v_{k} \in \mathbb{R}^{2 n}$ close enough to 0 , there is a holomorphic function $\lambda: \mathbb{D} \rightarrow M$, such that $\lambda(0)=v_{0}$ and $\frac{\partial^{l} \lambda}{\partial x^{l}}=v_{l}$, for $l=1, \ldots, k$. Moreover, we can choose $\lambda$ with $\mathcal{C}^{1}$ dependence on parameters $\left(v_{0}, \ldots, v_{k}\right) \in$ $\left(\mathbb{R}^{2 n}\right)^{k+1}$, where for holomorphic functions we consider $\mathcal{C}^{k^{\prime}}$ norm.

We can locally normalize coordinates with respect to a given holomorphic disc $\lambda$, that is we can assume that $\lambda(z)=(z, 0) \in \mathbb{C}^{n}$ and $J=J_{\text {st }}$ on $\mathbb{C} \times\{0\} \subset \mathbb{C}^{n}$, where $J_{\text {st }}$ is the standard almost complex structure in $\mathbb{C}^{n}$ (see section 1.2 in [1]) and moreover we can assume that for every $J$-holomorphic $\mu$ such that $\mu(0)=0$ we have $\Delta \mu(0)=0$ (see [8]).

An upper semi-continuous function $u$ on an open subset of $M$ is said to be $J$-plurisubharmonic or simpler plurisubharmonic, if a function $u \circ \lambda$ is subharmonic for every holomorphic function $\lambda$. We denote the set of plurisubharmonic functions on $\Omega \subset M$ by $\mathcal{P S H}(\Omega)$. For a smooth function $u$ it means that a matrix $\left(A_{p \bar{q}}\right)$ is nonnegative. Recently Harvey and Lawson proved that an upper semicontinuous locally integrable function $u$ is plurisubharmonic iff a current $i \partial \bar{\partial} u$ is positive. We say that a function $u \in \mathcal{C}^{1,1}(\Omega)$ is strictly plurisubharmonic if for every $K \Subset \Omega$ there is $m>0$ such that $\omega \leq i m \partial \bar{\partial} u$ a.e. in $K$, where $\omega$ is any hermitian metric ${ }^{4}$ on $\Omega$. If $u \in \mathcal{C}^{2}(\Omega)$ then the following conditions are equivalent:

(i) $u$ is strictly plurisubharmonic;

(ii) $i \partial \bar{\partial} u>0$;

(iii) $u$ is plurisubharmonic and $(i \partial \bar{\partial} u)^{n}>0$.

We say that a domain $\Omega \Subset M$ is strictly pseudoconvex of class $\mathcal{C}^{\infty}$ (respectively of class $\mathcal{C}^{1,1}$ ) if there is a strictly plurisubharmonic function $\rho$ of class $\mathcal{C}^{\infty}$ (respectively of class $\mathcal{C}^{1,1}$ ) in a neighbourhood of $\bar{\Omega}$, such that $\Omega=\{\rho<0\}$ and $\nabla \rho \neq 0$ on $\partial \Omega$. In that case we say that $\rho$ is a defining function for $\Omega$.

Let $z_{0} \in M$. The basic example of a (strictly) plurisubharmonic function in a neighbourhood of $z_{0}$ is $u(z)=\left(\operatorname{dist}\left(z, z_{0}\right)\right)^{2}$ (where dist is a distance in some Rimannian metric). Domains $\Omega_{\varepsilon}=\{u<\varepsilon\}$ are strictly pseudoconvex of class $\mathcal{C}^{\infty}$ for $\varepsilon>0$ small enough and they make a fundamental neighbourhood system for $z_{0}$.

4 Hermitian metric is a smooth positive $(1,1)$ form. 


\section{Comparison principle}

In this section $\Omega \Subset M$ is a domain not necessary strictly pseudoconvex but such that there is a bounded function $\rho \in \mathcal{C}^{2} \cap \mathcal{P S H}(\Omega)$.

In the pluripotential theory in $\mathbb{C}^{n}$, the comparison principle is a very effective tool. We give here the basic version for $J$-plurisubharmonic functions.

Proposition 2.1 (comparison principle) If $u, v \in \mathcal{C}^{2}(\bar{\Omega})$ are such that $u$ is a plurisubharmonic function, $(i \partial \bar{\partial} u)^{n} \geq(i \partial \bar{\partial} v)^{n}$ on the set $\{i \partial \bar{\partial} v>0\}$ and $u \leq v$ on $\partial \Omega$, then $u \leq v$ in $\bar{\Omega}$.

Proof First, let us assume that $(i \partial \bar{\partial} u)^{n}>(i \partial \bar{\partial} v)^{n}$ on the set $\{i \partial \bar{\partial} v \geq 0\}$ and a function $u-v$ takes its maximum in $z_{0} \in \Omega$. By Proposition 1.1 for small $\zeta \in T_{z_{0}}^{1,0} M$ there is a holomorphic disk $\lambda$ such that $\lambda(0)=z_{0}$ and $\frac{\partial \lambda}{\partial x}(0)-i J \frac{\partial \lambda}{\partial x}(0)=\zeta$. Hence at $z_{0}$

$$
\partial \bar{\partial}(v-u)(\zeta, \bar{\zeta})=\triangle((v-u) \circ \lambda)(0) \geq 0
$$

so we have $i \partial \bar{\partial} u \leq i \partial \bar{\partial} v$ and then we obtain $(i \partial \bar{\partial} u)^{n} \leq(i \partial \bar{\partial} v)^{n}$ which is the contradiction with our first assumption.

In the general case we put $u^{\prime}=u+\varepsilon\left(\rho-\sup _{\bar{\Omega}} \rho\right)$ and the lemma follows from the above case (with $u^{\prime}$ instead of $u$ ).

In Sect. 4 we use a slight stronger version of the proposition above.

Proposition 2.2 Suppose that $u, v \in \mathcal{C}^{2}(\bar{\Omega})$ are such that $u$ is a plurisubharmonic function and $(i \partial \bar{\partial} u)^{n} \geq(i \partial \bar{\partial} v)^{n}$ on the set

$$
\{i \partial \bar{\partial} v>0\} .
$$

Then for any $H \in \mathcal{P S H}$, an inequality

$$
\varlimsup_{z \rightarrow z_{0}}(u+H-v) \leq 0
$$

for any $z_{0} \in \partial \Omega$ implies $u+H \leq v$ on $\Omega$.

Proof Let $z_{0} \in \Omega$ be a point where a function $f=u+H-v$ attains a maximum and $\lambda$ is a holomorphic disk such that $\lambda(0)=z_{0}$. Because $H \circ \lambda$ is a subharmonic function one can find a sequence $t_{k}$ of nonzero complex numbers such that

$$
\lim _{k \rightarrow \infty} t_{k}=0
$$

and

$$
4 H \circ \lambda(0) \leq H \circ \lambda\left(t_{k}\right)+H \circ \lambda\left(i t_{k}\right)+H \circ \lambda\left(-t_{k}\right)+H \circ \lambda\left(-i t_{k}\right) .
$$

Hence

$$
\begin{aligned}
& \Delta((v-u) \circ \lambda)(0) \\
& \geq \varlimsup_{k \rightarrow \infty} \frac{4 f \circ \lambda(0)-f \circ \lambda\left(t_{k}\right)-f \circ \lambda\left(i t_{k}\right)-f \circ \lambda\left(-t_{k}\right)-f \circ \lambda\left(-i t_{k}\right)}{\left|t_{k}\right|^{2}} \geq 0 .
\end{aligned}
$$

Therefore we can obtain our result exactly as in the proof of the previous proposition. 


\section{A priori estimate}

In this section we will prove a $\mathcal{C}^{1,1}$ estimate for the smooth solution $u$ of the problem (2). By the general theory of elliptic equations (see for example [3]) we obtain from this the $\mathcal{C}^{k, \alpha}$ estimate and then the existence of a smooth solution. The uniqueness follows from the comparison principle.

Our proofs are close to these in [3] but more complicated because of the noncommutativity of some vector fields.

\subsection{Some technical preparation}

In this section we assume that $\Omega \Subset M$ is strictly pseudoconvex of class $\mathcal{C}^{\infty}$ with the defining function $\rho$. Let us fix a hermitian metric $\omega$ on $M$. From now all norms, gradient and hessian are taken with respect to this metric or more precisely with respect to a Rimannian metric which is given by $g(X, Y)=\omega(X, J Y)$ for vector fields $X, Y$.

Let $f \in \mathcal{C}^{\infty}(\bar{\Omega})$ be such that $d V=f \omega^{n}$. Then locally our Monge-Ampère equation $(i \partial \bar{\partial} u)^{n}=d V$ has a form:

$$
\operatorname{det}\left(A_{p \bar{q}}\right)=\tilde{f}=g f
$$

where $g=\operatorname{det}\left(-i \omega\left(\zeta_{p}, \bar{\zeta}_{q}\right)\right)$. So if vectors $\zeta_{1}, \ldots, \zeta_{n}$ are orthonormal (i.e. $\omega\left(\zeta_{p}, \bar{\zeta}_{q}\right)=$ $\left.i \delta_{p q}\right)$, then $g=1$.

The following elliptic operator is very useful

$$
L=L_{\zeta}=A^{p \bar{q}}\left(\zeta_{p} \bar{\zeta}_{q}-\left[\zeta_{p}, \bar{\zeta}_{q}\right]^{0,1}\right)
$$

Note that for $X, Y$ vector fields we have

$$
\begin{aligned}
X(\log \tilde{f}) & =A^{p \bar{q}} X A_{p \bar{q}}, \\
X Y(\log \tilde{f}) & =A^{p \bar{q}} X Y A_{p \bar{q}}-A^{p \bar{j}} A^{i \bar{q}}\left(Y A_{i \bar{j}}\right)\left(X A_{p \bar{q}}\right),
\end{aligned}
$$

where $\left(A^{p \bar{q}}\right)$ is the inverse of the matrix $\left(\overline{A_{p \bar{q}}}\right)$.

In the lemmas we specify exactly how a priori estimates depend on $\rho, f$ and $\varphi$. We should emphasize that they also depend strongly on $M, J, \omega, M^{\prime}$ and $m(\rho)$, where $M^{\prime}$ is some fixed domain such that $\Omega \Subset M^{\prime} \Subset M$ and $m(\rho)$ is defined as the smallest constant $m>0$ such that $\omega \leq m i \partial \bar{\partial} \rho$ on $\Omega$. The notion $C=C(A)$ really means that $C$ depends on an upper bound for $A$.

In the proofs below $C$ is a constant under control, but it can change from a line to a next line.

\subsection{Uniform estimate}

Lemma 3.1 We have $\|u\|_{L^{\infty}(\Omega)} \leq C$, where $C=C\left(\|\rho\|_{L^{\infty}(\Omega)},\|f\|_{L^{\infty}(\Omega)}\right.$, $\left.\|\varphi\|_{L^{\infty}(\Omega)}\right)$.

Proof From the comparison principle and the maximum principle we have

$$
\left\|f^{1 / n}\right\|_{L^{\infty}(\Omega)} m(\rho) \rho+\inf _{\partial \Omega} \varphi \leq u \leq \sup _{\partial \Omega} \varphi .
$$




\subsection{Gradient estimate}

In the next two lemmas we shall prove a priori estimate for the first derivative.

Lemma 3.2 We have

$$
\|u\|_{\mathcal{C}^{0,1}(\partial \Omega)} \leq C
$$

where $C=C\left(\|\rho\|_{\mathcal{C}^{0,1}(\Omega)},\|f\|_{L^{\infty}(\Omega)},\|\varphi\|_{\mathcal{C}^{1,1}(\Omega)}\right)$.

Proof We can choose $A>0$ such that $A i \partial \bar{\partial} \rho+i \partial \bar{\partial} \varphi \geq f^{1 / n} \omega$ and $A i \partial \bar{\partial} \rho \geq i \partial \bar{\partial} \varphi$. Thus by the comparison principle and the maximum principle we have

$$
\varphi+A \rho \leq u \leq \varphi-A \rho
$$

for $A$ large enough. So on the boundary we have

$$
|\nabla u| \leq|\nabla A \rho|+|\nabla \varphi| \text {. }
$$

\section{Lemma 3.3 We have}

$$
\|u\|_{\mathcal{C}^{0,1}(\Omega)} \leq C
$$

where $C=C\left(\|\rho\|_{\mathcal{C}^{0,1}(\Omega)},\left\|f^{1 / n}\right\|_{\mathcal{C}^{0,1}},\|u\|_{\mathcal{C}^{0,1}(\partial \Omega)}\right)$.

Proof Let us consider the function $v=\psi|\nabla u|^{2}$, where a smooth plurisubharmonic function $\psi$ will be determined later. Let us assume that $v$ takes its maximum in $z_{0} \in \Omega$. We can choose $\zeta_{1}, \ldots, \zeta_{n}$, such that they are orthonormal in a neighbourhood of $z_{0}$, and the matrix $A_{p \bar{q}}$ is diagonal at $z_{0}$. From now on all formulas are assumed to hold at $z_{0}$.

We have $X v=0$, hence $X\left(|\nabla|^{2}\right)=-|\nabla u|^{2} X \log \psi$. We can calculate

$$
\begin{aligned}
L(v)= & L(\psi)|\nabla u|^{2}+\psi L\left(|\nabla u|^{2}\right)+A^{p \bar{p}}\left(\psi_{p}\left(|\nabla u|^{2}\right)_{\bar{p}}+\psi_{\bar{p}}\left(|\nabla u|^{2}\right)_{p}\right) \\
= & |\nabla u|^{2} A^{p \bar{p}}\left(\psi_{p \bar{p}}-\left[\zeta_{p}, \overline{\zeta_{p}}\right]^{0,1} \psi-2 \frac{\left|\psi_{p}\right|^{2}}{\psi}\right)+\psi L\left(|\nabla u|^{2}\right), \\
L\left(|\nabla u|^{2}\right)= & A^{p \bar{p}}\left(\left(|\nabla u|^{2}\right)_{p \bar{p}}-\left[\zeta_{p}, \overline{\zeta_{p}}\right]^{0,1}|\nabla u|^{2}\right) \\
= & A^{p \bar{p}} \sum_{k}\left(u_{p \bar{p} k} u_{\bar{k}}+u_{k} u_{p \bar{p} \bar{k}}+\left|u_{p k}\right|^{2}+\left|u_{\bar{p} k}\right|^{2}\right. \\
& \left.-\left[\zeta_{p}, \overline{\zeta_{p}}\right]^{0,1} u_{k} u_{\bar{k}}-u_{k}\left[\zeta_{p}, \overline{\zeta_{p}}\right]^{0,1} u_{\bar{k}}\right), \\
& A^{p \bar{p}}\left(u_{p \bar{p} k}-\left[\zeta_{p}, \overline{\zeta_{p}}\right]^{0,1} u_{k}\right) \\
= & A^{p \bar{p}}\left(u_{k p \bar{p}}-\zeta_{k}\left[\zeta_{p}, \overline{\zeta_{p}}\right]^{0,1} u+\zeta_{p}\left[\bar{\zeta}_{p}, \zeta_{k}\right] u+\left[\zeta_{p}, \zeta_{k}\right] \overline{\zeta_{p}} u\right) \\
= & (\log f)_{k}+A^{p \bar{p}}\left(\zeta_{p}\left[\overline{\zeta_{p}}, \zeta_{k}\right] u+\bar{\zeta}_{p}\left[\zeta_{p}, \zeta_{k}\right] u+\left[\left[\zeta_{p}, \zeta_{k}\right], \overline{\zeta_{p}}\right] u\right. \\
& \left.-\left[\left[\zeta_{p}, \overline{\zeta_{p}}\right]^{0,1}, \zeta_{k}\right] u\right) .
\end{aligned}
$$

Then we have

$$
\begin{aligned}
& \left|A^{p \bar{p}}\left(u_{p \bar{p} k}-\left[\zeta_{p}, \bar{\zeta}_{p}\right]^{0,1} u_{k}\right)\right| \\
& \quad \leq C\left(\frac{\left\|f^{1 / n}\right\|_{\mathcal{C}^{0,1}}}{f^{1 / n}}+A^{p \bar{p}}\left(\sum_{s}\left(\left|u_{p s}\right|+\left|u_{p \bar{s}}\right|\right)+|\nabla u|\right)\right)
\end{aligned}
$$


and similarly

$$
\begin{aligned}
& \left|A^{p \bar{p}}\left(u_{p \bar{p} \bar{k}}-\left[\zeta_{p}, \bar{\zeta}_{p}\right]^{0,1} u_{\bar{k}}\right)\right| \\
& \quad \leq C\left(\frac{\left\|f^{1 / n}\right\|_{\mathcal{C}^{0,1}}}{f^{1 / n}}+A^{p \bar{p}}\left(\sum_{s}\left(\left|u_{p s}\right|+\left|u_{p \bar{s}}\right|\right)+|\nabla u|\right)\right)
\end{aligned}
$$

so for the proper choice of $\psi$ (we can get $\psi=e^{A \rho}+B$ for $A, B$ large enough) we have $L(v)(0)>0$ and this is a contradiction with the maximality of $v$.

\section{$3.4 \mathcal{C}^{1,1}$ estimate}

Let us fix a point $P \in \partial \Omega$. Now we give the $\mathcal{C}^{1,1}$ estimate in a point $P$ (which does not depend on $P$ ). The estimate of $X Y u(P)$, where $X, Y$ are tangent to $\partial \Omega$, follows from the gradient estimate.

Lemma 3.4 Let $N \in T_{P} M$ be orthogonal to $\partial \Omega$ such that $N \rho=-1$ and let $X$ be a vector field on a neighbourhood of $P$ tangent to $\partial \Omega$ on $\partial \Omega$. We have

$$
|N X u(P)| \leq C,
$$

where $C=C\left(\|\rho\|_{\mathcal{C}^{0,1}(\Omega)},\left\|f^{1 / n}\right\|_{\mathcal{C}^{0,1}},\|\varphi\|_{\mathcal{C}^{2,1}(\Omega)},\|X\|_{\mathcal{C}^{0,1}},\|u\|_{\mathcal{C}^{0,1}(\Omega)}\right)$.

Proof Let $X_{1}, X_{2}, \ldots, X_{n}$ be (real) vector fields on $U$ a neighbourhood of $P$, tangent at $P$ to $\partial \Omega$, such that $X_{1}, J X_{1}, \ldots, X_{n}, J X_{n}$ is a frame. Consider the function

$$
v=X(u-\varphi)+B \rho+\sum_{k=1}^{n}\left|X_{k}(u-\varphi)\right|^{2}-A(\operatorname{dist}(P, \cdot))^{2} .
$$

Let $V \Subset U$ be a neighbourhood of $P$ and $S=V \cap \Omega$. For $A$ large enough $v \leq 0$ on $\partial S$.

Our goal is to show that for $B$ large enough we have $v \leq 0$ on $\bar{S}$. Let $z_{0} \in S$ be a point where $v$ attains a maximum and let $\zeta_{1}, \ldots, \zeta_{n}$ be orthonormal and such that $\left(A_{p \bar{q}}\right)$ is diagonal. From now on all formulas are assumed to hold at $z_{0}$. Let us calculate:

$$
m(\rho) L(\rho) \geq \sum A^{p \bar{p}}
$$

and

$$
L\left(-X \varphi-A(\operatorname{dist}(P, \cdot))^{2}\right) \geq-C \sum A^{p \bar{p}},
$$

hence for $B$ large enough

$$
L\left(B \rho-X \varphi-A(\operatorname{dist}(P, \cdot))^{2}\right) \geq \frac{B}{2 m(\rho)} \sum A^{p \bar{p}} .
$$

To estimate $L\left(X u+\sum_{k=1}^{n}\left|X_{k}(u-\varphi)\right|^{2}\right)$ let us first consider $Y \in\left\{X, X_{1}, \ldots X_{n}\right\}$ and calculate

$$
\begin{aligned}
L(Y u) & =A^{p \bar{q}}\left(\zeta_{p} \bar{\zeta}_{q} Y u-\left[\zeta_{p}, \bar{\zeta}_{q}\right]^{0,1} Y u\right) \\
& =Y \log f+A^{p \bar{q}}\left(\zeta_{p}\left[\bar{\zeta}_{q}, Y\right] u+\left[\zeta_{p}, Y\right] \bar{\zeta}_{q} u-\left[\left[\zeta_{p}, \bar{\zeta}_{q}\right]^{0,1}, Y\right] u\right) .
\end{aligned}
$$


There are $\alpha_{q, k}, \beta_{q, k} \in \mathbb{C}$ such that

$$
\left[\bar{\zeta}_{q}, Y\right]=\sum_{k=1}^{n} \alpha_{q, k} \bar{\zeta}_{k}+\beta_{q, k} X_{k}
$$

and so

$$
A^{p \bar{q}} \zeta_{p}\left[\bar{\zeta}_{q}, Y\right] u=\sum_{q} \alpha_{q, q}+\sum_{k=1}^{n} A^{p \bar{p}} \beta_{p, k} \zeta_{p} X_{k} u+A^{p \bar{p}} Z_{p} u
$$

where $Z_{p}$ are vector fields under control. This gives us

$$
\left|A^{p \bar{q}} \zeta_{p}\left[\bar{\zeta}_{q}, Y\right] u\right| \leq C A^{p \bar{p}}\left(1+\sum_{k}\left|\beta_{p, k} \zeta_{p} X_{k} u\right|\right) .
$$

In a similar way we can estimate $A^{p \bar{q}}\left[\zeta_{p}, Y\right] \bar{\zeta}_{q} u$ and we obtain

$$
|L(Y u)| \leq C A^{p \bar{p}}\left(1+\sum_{k}\left|\zeta_{p} X_{k} u\right|\right) .
$$

Therefore

$$
\begin{aligned}
& L\left(X u+\sum_{k}\left|X_{k}(u-\varphi)\right|^{2}\right) \\
& \geq A^{p \bar{p}} \sum_{k=1}^{n}\left(\zeta_{p} X_{k}(u-\varphi)\right)\left(\bar{\zeta}_{p} X_{k}(u-\varphi)\right)-C A^{p \bar{p}}\left(1+\sum_{k}\left|\zeta_{p} X_{k} u\right|\right) \\
& \geq A^{p \bar{p}} \sum_{k}\left|\zeta_{p} X_{k} u\right|^{2}-C A^{p \bar{p}}\left(1+\sum_{k}\left|\zeta_{p} X_{k} u\right|\right) .
\end{aligned}
$$

Now for $B$ large enough, since $L(v)\left(z_{0}\right)>0$, we have contradiction with maximality of $v$. Hence $v \leq 0$ on $S$ and so $N X u(P) \leq C$.

Lemma 3.5 Let $X$ be a vector field orthogonal to $\partial \Omega$ at $P$. We have

$$
\|X X u(P)\| \leq C,
$$

where

$$
C=C\left(\|\rho\|_{\mathcal{C}^{2,1}(\Omega)},\left\|f^{1 / n}\right\|_{\mathcal{C}^{0,1}},\left\|f^{-1}\right\|_{L^{\infty}(\Omega)},\|\varphi\|_{\mathcal{C}^{3,1}(\Omega)},\|X\|_{\mathcal{C}^{0,1}},\|u\|_{\mathcal{C}^{0,1}(\Omega)}\right) .
$$

Proof By the previous Lemma it is enough to prove that

$$
|\zeta|^{2} \leq C\left(\zeta \bar{\zeta}-[\zeta, \bar{\zeta}]^{0,1}\right) u(P)
$$

for every vector field $\zeta \in T^{1,0} M$ tangent (at $P$ ) to $\partial \Omega$.

Because our argue are local we can assume that $P=0 \in \mathbb{C}^{n}$. Let $\zeta_{1}, \zeta_{2}, \ldots \zeta_{n} \in T^{1,0}$ be an orthonormal frame in a neighbourhood of 0 such that $\zeta_{k} \rho=-\delta_{k n}$. We can assume that $\zeta_{1}=\zeta$. By the strictly pseudoconvexity we have $\left(\zeta \bar{\zeta}-[\zeta, \bar{\zeta}]^{0,1}\right) \rho(P) \neq 0$, so we can also assume that $\left(\zeta \bar{\zeta}-[\zeta, \bar{\zeta}]^{0,1}\right) \varphi(P)=0$.

From the strictly pseudoconvexity and using the Proposition 1.1 (for $k=2$ ) we can choose $J$-holomorphic disk $\lambda$ such that $\lambda(0)=0, \frac{\partial \lambda}{\partial z}(0)=a \zeta$ and 


$$
\rho \circ \lambda(z)=b|z|^{2}+O\left(|z|^{3}\right)
$$

for some $a, b>0$. In particular we have

$$
|z|^{2} \leq C \operatorname{dist}(\lambda(z), \bar{\Omega})
$$

Indeed, for $a>0$ small enough, by the proposition 1.1 (for $k=1$ ) there is a $J$-holomorphic $\operatorname{disk} \tilde{\lambda}$ such that $\tilde{\lambda}(0)=0$ and $\frac{\partial \tilde{\lambda}}{\partial z}(0)=a \zeta$. Then (by changing coordinates) we can assume $(\zeta J)(0)=0$, so for any $J$-holomorphic disk $\lambda$ such that $\lambda(0)=0$ and $\frac{\partial \lambda}{\partial z}(0)=a \zeta$ we have $\frac{\partial^{2} \lambda}{\partial x^{2}}(0)=-J \frac{\partial^{2} \lambda}{\partial x \partial y}(0)=-\frac{\partial^{2} \lambda}{\partial y^{2}}(0)$. Now if we put

$$
\frac{\partial^{2} \lambda}{\partial x^{2}}(0)=a^{2}\left(0,4 \frac{\partial^{2} \rho}{\partial x_{1}^{2}}(0)-2\left(\zeta \bar{\zeta}-[\zeta, \bar{\zeta}]^{0,1}\right) \rho(0),-4 \frac{\partial^{2} \rho}{\partial x_{1} \partial x_{2}}(0)\right)
$$

we obtain (3.1) with $b=a^{2}\left(\zeta \bar{\zeta}-[\zeta, \bar{\zeta}]^{0,1}\right) \rho(0)$.

Once again changing coordinates we may assume $\lambda\left(z_{1}\right)=\left(z_{1}, 0\right), \zeta_{k}(0)=\frac{\partial}{\partial z_{k}}$ for $k=1, \ldots, n$ and for every $J$-holomorphic disk $\mu$ such that $\mu(0)=0$ we have

$$
\frac{\partial^{2} \mu}{\partial z \partial \bar{z}}(0)=0
$$

We can find a holomorphic cubic polynomial $p_{1}$ and a complex number $\alpha$ such that

$$
\begin{aligned}
\varphi(z)= & \varphi(0)+\varphi^{\prime}(0)(z) \\
& +\operatorname{Re}\left(\sum_{p=1}^{n} \frac{\partial^{2} \varphi}{\partial z_{1} \partial \bar{z}_{p}} z_{1} \bar{z}_{p}+p_{1}(z)+\alpha z_{1}\left|z_{1}\right|^{2}\right)+O\left(\left|z_{1}\right|^{4}+\left|z_{2}\right|^{2}+\ldots+\left|z_{n}\right|^{2}\right) .
\end{aligned}
$$

By (3.2) we can choose another cubic polynomial $p_{2}$ and numbers $\beta_{1}, \beta_{2}, \ldots, \beta_{n} \in \mathbb{C}, \beta_{1}>$ 0 such that

$$
\operatorname{Re} z_{n}=\operatorname{Re}\left(\sum_{p=1}^{n} \beta_{p} z_{1} \bar{z}_{p}+p_{2}(z)\right)+O\left(\left|z_{1}\right|^{3}+\left|z_{2}\right|^{2}+\ldots+\left|z_{n}\right|^{2}\right) \text { on } \partial \Omega .
$$

Then we obtain

$$
\varphi(z)+\varphi(0)=\varphi^{\prime}(0)(z)+\operatorname{Re}\left(\sum_{p=2}^{n} a_{p} z_{1} \bar{z}_{p}+p_{3}(z)\right)+O\left(\left|z_{2}\right|^{2}+\ldots+\left|z_{n}\right|^{2}\right)
$$

for some numbers $a_{2}, \ldots, a_{n} \in \mathbb{C}$ and a new cubic polynomial $p_{3}$. Hence

$$
u(z)-u(0)=\operatorname{Re}\left(\sum_{p=2}^{n} a_{p} z_{1} \bar{z}_{p}+p_{4}(z)\right)+O\left(\left|z_{2}\right|^{2}+\ldots+\left|z_{n}\right|^{2}\right)
$$

for $z \in \partial \Omega$ and same polynomial $p_{4}$.

Let $B>1^{5}$ and $D=B^{-1} \max \left\{\left|a_{2}\right|, \ldots,\left|a_{n}\right|\right\}$. By the Proposition 1.1 (again for $k=2$ ) there is a family of $J$-holomorphic disks $g_{w}: \mathbb{D} \rightarrow \mathbb{C}^{n}, w \in \mathbb{C}^{n-1}, g_{w}=\left(g_{w}^{1}, \ldots, g_{w}^{n}\right)$ such that

5 Constants $C$ below do not depend on the constant $B$. 


$$
\begin{aligned}
g_{w}(0) & =(0, w), \\
\frac{\partial g_{w}}{\partial z}(0) & =\left(1,-\frac{a_{2}}{B}, \ldots,-\frac{a_{n}}{B}\right), \\
\left\|g_{w}-\lambda\right\|_{\mathcal{C}^{4}} & \leq C(|w|+D)
\end{aligned}
$$

and a function $G: \mathbb{D} \times \mathbb{C}^{n-1} \rightarrow \mathbb{C}^{n}$ given by $G(z, w)=g_{w}(z)$ is of class $\mathcal{C}^{4}$. Then we have

$$
\left|g_{w}(z)-\left(w_{1}+z, w_{2}-\frac{a_{2} z}{B}, \ldots, w_{n}-\frac{a_{n} z}{B}\right)\right|<C|z|^{2}(|w|+D)
$$

for $z \in \mathbb{D}$ and by (3.2)

$$
|z|<C(\sqrt{|w|}+D)
$$

if $g_{w}(z) \in \Omega$.

We can choose domains $U \subset \mathbb{D}, V \subset \mathbb{C}^{n-1}, W \subset \mathbb{C}^{n}$ such that $0 \in W, G(\partial U \times V) \cap \bar{\Omega}=$ $\emptyset$ and $\left.G\right|_{U \times V}$ is a diffeomorphism onto $W$.

Let

$$
h\left(g_{w}(z)\right)=\operatorname{Re} p_{w}(z)+A B|w|^{2}+\varepsilon \rho,
$$

where $A, \varepsilon>0$ and $p_{w}$ is a holomorphic cubic polynomial in one variable such that

$$
\operatorname{Re} p_{4}\left(g_{w}(z)\right)=\operatorname{Re} p_{w}(z)+a_{w}|z|^{2}+\operatorname{Re} b_{w} z|z|^{2}+O\left(|z|^{4}\right)
$$

for some $a_{w} \in \mathbb{R}, b_{w} \in \mathbb{C}$. Note that $\left|b_{w}\right|<C(|w|+D)$ and by (3.3) $\left|a_{w}\right|<C|w|$. Thus enlarging $A$ (if necessary) and using also (3.6) we obtain

$$
\operatorname{Re} p_{4}\left(g_{w}(z)\right) \leq h\left(g_{w}(z)\right)+\frac{1}{2} D^{2}|z|^{2}
$$

on $\partial \Omega$.

By inequalities (3.5) and (3.6) we have

$$
\begin{aligned}
& 2 \sum_{k=2}^{n} \operatorname{Re} a_{k} g_{w}^{1}(z) g_{w}^{k}(z) \\
& \quad=\sum_{k=2}^{n} B\left(\left|-\frac{a_{k} g_{w}^{1}(z)}{B}-g_{w}^{k}(z)\right|^{2}-\left|\frac{a_{k} g_{w}^{1}(z)}{B}\right|^{2}-\left|g_{w}^{k}(z)\right|^{2}\right) \\
& \quad \leq B\left(|w|^{2}-D^{2}|z|^{2}+C|z|^{4}\left(|w|^{2}+D^{2}\right)\right) \leq C B|w|^{2}-\frac{1}{2} D^{2}|z|^{2}
\end{aligned}
$$

for $B$ large enough. By an above estimate, (3.4), and (3.7) we obtain that if $A$ is large enough then $h \geq u-u(0)$ on $\partial \Omega \cap W$. Again enlarging $A$ we can assume $h \geq u-u(0)$ on $\partial S$ where $S=\Omega \cap W$. Since $i \partial \bar{\partial} h$ is under control for $\varepsilon$ enough small we get an inequality

$$
(i \partial \bar{\partial} h)^{n}<(i \partial \bar{\partial} u)^{n}
$$

on the set $S \cap\{i \partial \bar{\partial} h>0\}$. This by the comparison principle gives us $h \geq u-u(0)$ on $S$. Note that $h_{N} \geq u_{N}, \varphi_{1 \overline{1}}=0$ and $\varphi_{N}=h_{N}-\varepsilon \rho_{N}=h_{N}+\varepsilon$, so we can conclude that

$$
u_{1 \overline{1}}=u_{1 \overline{1}}-\varphi_{1 \overline{1}}=\left(\varphi_{N}-u_{N}\right) \rho_{1 \overline{1}} \geq \varepsilon \rho_{1 \overline{1}} .
$$

Finally we will obtain the interior $\mathcal{C}^{1,1}$ estimate, which together with previous lemmas gives us a full $\mathcal{C}^{1,1}$ estimate. By a standard argumentation this ends the proof of Theorem 1. 
Lemma 3.6 We have

$$
\|H u\|_{L^{\infty}(\Omega)} \leq C
$$

where $\mathrm{Hu}$ is a Hessian of $\mathrm{u}$ and

$$
C=C\left(\|\rho\|_{\mathcal{C}^{0,1}(\Omega)},\left\|f^{\frac{1}{2 n}}\right\|_{\mathcal{C}^{1,1}},\|u\|_{\mathcal{C}^{0,1}(\Omega)},\|H u\|_{L^{\infty}(\partial \Omega)}\right) .
$$

Proof Let us define $M$ as the biggest eigenvalue of the Hessian $H u$. We will show that the function

$$
\Lambda=\psi e^{K|\nabla u|^{2}} M
$$

where a smooth plurisubharmonic function $\psi>1$ and a small positive number $K$ will be determined later, does not attain maximum in $\Omega$. Because a function $u$ is plurisubharmonic this will give (3.8).

Assume that a maximum of the function $\Lambda$ is attained at a point $z_{0} \in \Omega$ (otherwise we are done). There are $\zeta_{1}, \ldots, \zeta_{n} \in T_{z_{0}}^{1,0} M$ orthonormal at $z_{0}$ such that the matrix $\left(A_{p \bar{q}}\right)$ is diagonal at $z_{0}$. Let $X \in T_{z_{0}} M$ be such that $\|X\|=1$ and $M=H(X, X)$. We can normalize coordinates near $z_{0}$ such that $z_{0}=0 \in \mathbb{C}^{n}, X=\frac{\partial}{\partial x_{1}}(0)$ and $J(z, 0)=J_{s t}$ for small $z \in \mathbb{C}$. Let us extend $X$ as $\frac{\partial}{\partial x_{1}}$ and then in a natural way we can extend $\zeta_{1}, \ldots, \zeta_{n}$ to some neighbourhood $U$ of 0 such that $\left[\zeta_{k}, X\right](0)=0$ and $\left[\zeta_{k}, \bar{\zeta}_{k}\right](0)=0$ for $k=1, \ldots, n$. Indeed, on $U \cap \mathbb{C} \times\{0\}$ we can put $\zeta_{k}$ as the same linear combination of vectors $\frac{\partial}{\partial z_{1}} \ldots, \frac{\partial}{\partial z_{n}}$ as in 0 . Then for some small $a>0$ we can take (for $\zeta_{k}$ not tangent to $\mathbb{C} \times\{0\}$ ) $J$-holomorphic disks $d_{k}: \mathbb{D} \rightarrow U$ such that $d_{k}(0)=0$ and $\frac{\partial d_{k}}{\partial z}(0)=a \zeta_{k}(0)$, and on the image of $d_{k}$ we can put $\zeta_{k}(w)=a^{-1} \frac{\partial d_{k}}{\partial z}\left(d_{k}^{-1}(w)\right)$. On the end we extend the vector fields on whole $U$.

Let

$$
v=\psi e^{K|\nabla u|^{2}} \frac{H u\left(\frac{\partial}{\partial x_{1}}, \frac{\partial}{\partial x_{1}}\right)}{\left|\frac{\partial}{\partial x_{1}}\right|^{2}}=\Psi e^{K|\nabla u|^{2}}\left(u_{x_{1} x_{1}}+T u\right) \text { on } U,
$$

where $\Psi=\frac{\psi}{\left|\frac{\partial}{\partial x_{1}}\right|^{2}}$ and $T$ is a vector field (which is under control), then also a function $v$ has a maximum at 0 (in particular $L(v) \leq 0$ ). Let us put $\mu=u_{x_{1} x_{1}}+T u$ (then $\left|\frac{\partial}{\partial x_{1}}(0)\right|^{2} \mu(0)=$ $M(0)$ ). Note that we have $X Y u \leq C \mu$ for vector fields $X, Y$ (which are under control). Assume $\mu>1$ (otherwise we have $\Lambda<C$, so we are done).

From now all formulas are assumed to hold at 0 . We estimate $L(v)$ from below:

$$
L(v)=L\left(\Psi e^{K|\nabla u|^{2}}\right) \mu+\Psi e^{K|\nabla u|^{2}} L(\mu)-2 A^{p \bar{p}} \frac{\left(\Psi e^{K|\nabla u|^{2}}\right)_{p}\left(\Psi e^{K|\nabla u|^{2}}\right)_{\bar{p}} \mu}{\Psi e^{K|\nabla u|^{2}}}
$$

To estimate the first term let us calculate

$$
\begin{aligned}
L( & \left.\Psi e^{K|\nabla u|^{2}}\right) \\
= & e^{K|\nabla u|^{2}} A^{p \bar{p}}\left(\Psi_{p \bar{p}}+2 K \operatorname{Re}\left(\Psi_{p}\left(|\nabla u|^{2}\right)_{\bar{p}}\right)+K \Psi\left(|\nabla u|^{2}\right)_{p \bar{p}}\right. \\
& \left.+K^{2} \Psi\left|\left(|\nabla u|^{2}\right)_{p}\right|^{2}\right), A^{p \bar{p}}\left(|\nabla u|^{2}\right)_{p \bar{p}} \\
= & A^{p \bar{p}} \sum_{k}\left(\left(\zeta_{p} \bar{\zeta}_{p} \eta_{k} u\right) u_{\bar{k}}+u_{k}\left(\zeta_{p} \bar{\zeta}_{p} \bar{\eta}_{k} u\right)+\left(\bar{\zeta}_{p} \eta_{k} u\right)\left(\zeta_{p} \bar{\eta}_{k} u\right)+\left(\zeta_{p} \eta_{k} u\right)\left(\bar{\zeta}_{p} \bar{\eta}_{k} u\right)\right)
\end{aligned}
$$




$$
\begin{aligned}
& =\sum_{k}\left((\log \tilde{f})_{k} u_{\bar{k}}+(\log \tilde{f})_{\bar{k}} u_{k}\right) \\
& +A^{p \bar{p}} \sum_{k}\left(\left(\zeta_{p}\left[\zeta_{\bar{p}}, \eta_{k}\right] u\right) u_{\bar{k}}+\left(\left[\zeta_{p}, \eta_{k}\right] u_{\bar{p}}\right) u_{\bar{k}}+\left(\zeta_{p}\left[\bar{\zeta}_{p}, \bar{\eta}_{k}\right] u\right) u_{k}+\left(\left[\zeta_{p}, \bar{\eta}_{k}\right] u_{\bar{p}}\right) u_{k}\right) \\
& +A^{p \bar{p}} \sum_{k}\left(2 K \operatorname{Re}\left(\eta_{k}\left[\zeta_{p}, \bar{\zeta}_{p}\right]^{0,1} u u_{\bar{k}}\right)+\left(\bar{\zeta}_{p} \eta_{k} u\right)\left(\zeta_{p} \bar{\eta}_{k} u\right)+\left(\zeta_{p} \eta_{k} u\right)\left(\bar{\zeta}_{p} \bar{\eta}_{k} u\right)\right)
\end{aligned}
$$

where $\eta_{1}, \ldots \eta_{n}$ is an orthonormal frame such that $\eta_{k}(0)=\zeta_{k}(0)$. Therefore we have

$$
A^{p \bar{p}}\left(|\nabla u|^{2}\right)_{p \bar{p}} \geq-C+A^{p \bar{p}} \frac{1}{2} \sum_{k}\left(\left(\bar{\zeta}_{p} \zeta_{k} u\right)\left(\zeta_{p} \bar{\zeta}_{k} u\right)+\left(\zeta_{p} \zeta_{k} u\right)\left(\bar{\zeta}_{p} \bar{\zeta}_{k} u\right)-C\right),
$$

hence

$$
\begin{aligned}
L\left(\Psi e^{K|\nabla u|^{2}}\right) \geq & -C e^{K|\nabla u|^{2}} \\
& +e^{K|\nabla u|^{2}} A^{p \bar{p}}\left(\Psi_{p \bar{p}}-C K\left|\Psi_{p}\right| \sum_{k}\left(\left|u_{p \bar{k}}\right|+\left|u_{p k}\right|+1\right)\right) \\
& +e^{K|\nabla u|^{2}} A^{p \bar{p}} \Psi\left(\frac{1}{2} K-C K^{2}\right) \sum_{k}\left(\left|u_{p \bar{k}}\right|^{2}+\left|u_{p k}\right|^{2}\right) .
\end{aligned}
$$

Let us start the calculation for the second term

$$
\begin{aligned}
L(\mu)= & L\left(u_{x_{1} x_{1}}\right)+L(T u), \\
L(T u) \leq & T(\log \tilde{f})-C(\mu+1) \sum A^{p \bar{p}}, \\
L\left(u_{x_{1} x_{1}}\right)= & (\log \tilde{f})_{x_{1} x_{1}}+A^{p \bar{p}} A^{q \bar{q}}\left|X\left(\zeta_{p} \bar{\zeta}_{q}-\left[\zeta_{p}, \bar{\zeta}_{q}\right]^{0,1}\right) u\right|^{2} \\
& +A^{p \bar{p}}\left(\zeta_{p}\left[\bar{\zeta}_{p}, X\right] X u+\left[\zeta_{p}, X\right] \bar{\zeta}_{p} X u+X \zeta_{p}\left[\bar{\zeta}_{p}, X\right] u+X\left[\zeta_{p}, X\right] \bar{\zeta}_{p} u\right. \\
& \left.+X X\left[\zeta_{p}, \bar{\zeta}_{p}\right]^{0,1} u\right) \\
= & (\log \tilde{f})_{x_{1} x_{1}}+A^{p \bar{p}} A^{q \bar{q}}\left|X\left(\zeta_{p} \bar{\zeta}_{q}-\left[\zeta_{p}, \bar{\zeta}_{q}\right]^{0,1}\right) u\right|^{2} \\
& +A^{p \bar{p}}\left(\left[\zeta_{p},\left[\bar{\zeta}_{p}, X\right]\right] X u+X\left[\zeta_{p},\left[\bar{\zeta}_{p}, X\right]\right] u+\left[X,\left[\bar{\zeta}_{p}, X\right]\right] \zeta_{p} u\right. \\
& \left.+\left[X,\left[\zeta_{p}, X\right]\right] \bar{\zeta}_{p} u\right)+A^{p \bar{p}}\left(X\left[X,\left[\zeta_{p}, \bar{\zeta}_{p}\right]^{0,1}\right] u+\left[X,\left[\zeta_{p}, \bar{\zeta}_{p}\right]^{0,1}\right] X u\right) \\
\geq & -C(\mu+1) \sum A^{p \bar{p}}
\end{aligned}
$$

and we obtain

$$
L(\mu) \geq-C \mu \sum A^{p \bar{p}} .
$$

Now we come to the last term

$$
\begin{aligned}
& -2 A^{p \bar{p}} \frac{\left(\Psi e^{K|\nabla u|^{2}}\right)_{p}\left(\Psi e^{K|\nabla u|^{2}}\right)_{\bar{p}}}{\Psi e^{K|\nabla u|^{2}}} \\
& =-2 A^{p \bar{p}} e^{K|\nabla u|^{2}}\left(\frac{\left|\Psi_{p}\right|^{2}}{\Psi}+2 K \operatorname{Re}\left(\Psi_{p}\left(|\nabla u|^{2}\right)_{\bar{p}}\right)+K^{2} \Psi\left(|\nabla u|^{2}\right)_{\bar{p}}\left(|\nabla u|^{2}\right)_{p}\right) \\
& \geq-2 e^{K|\nabla u|^{2}} A^{p \bar{p}}\left(\frac{\left|\Psi_{p}\right|^{2}}{\Psi}+C K\left|\Psi_{p}\right| \sum_{k}\left(\left|u_{p \bar{k}}\right|+\left|u_{p k}\right|\right)+K^{2} \sum_{k}\left(\left|u_{p \bar{k}}\right|^{2}+\left|u_{p k}\right|^{2}\right)\right) .
\end{aligned}
$$


Therefore there is a constant $C_{0}>1$ which is under control such that

$$
\begin{aligned}
L(v) \geq & \mu e^{K|\nabla u|^{2}} A^{p \bar{p}}\left(\Psi_{p \bar{p}}+\Psi\left(\frac{1}{2} K-C_{0} K^{2}\right) \sum_{k}\left(\left|u_{p \bar{k}}\right|^{2}+\left|u_{p k}\right|^{2}\right)-2 \frac{\left|\Psi_{p}\right|^{2}}{\Psi}\right) \\
& -C_{0} \mu e^{K|\nabla u|^{2}} A^{p \bar{p}}(1+K)\left(1+\left|\Psi_{p}\right|\right)\left(1+\sum_{k}\left(\left|u_{p \bar{k}}\right|+\left|u_{p k}\right|\right)\right) .
\end{aligned}
$$

For a proper choice of $K$ and $\psi\left(K=\frac{1}{2 C_{0}}, \psi=e^{A \phi}+4 e^{A}\right.$ where $\frac{1}{2}<\phi<1$ is strictly plurisubharmonic in a neighborhood of $\bar{\Omega}$ and $A$ is large enough) we can conclude that $L(v)>0$ and this is a contradiction with the maximality of $v$.

\section{Maximal plurisubharmonic functions}

We say that a function $u \in \mathcal{P S H}(\Omega)$ is maximal if for every function $v \in \mathcal{P S H}(\Omega)$ such that $v \leq u$ outside a compact subset of $\Omega$ we have $v \leq u$ in $\Omega$.

Now we want to find the solution to the following Dirichlet problem:

$$
\left\{\begin{array}{l}
u \in \mathcal{P} \mathcal{S H}(\Omega) \cap \mathcal{C}(\bar{\Omega}) \\
u \text { is maximal } \\
u=\varphi \text { on } \partial \Omega
\end{array}\right.
$$

where $\Omega$ is a strictly pseudoconvex domain of class $\mathcal{C}^{1,1}$ and $\varphi$ is a continuous function on $\partial \Omega$.

Proposition 4.1 If $\varphi \in \mathcal{C}^{1,1}(\bar{\Omega})$, then there is a unique solution $u \in \mathcal{C}^{0,1}(\bar{\Omega})$ of the problem (4.1) and

$$
\|u\|_{\mathcal{C}^{0,1}(\bar{\Omega})} \leq C=C\left(\|\rho\|_{\mathcal{C}^{0,1}(\Omega)}, m(\rho),\|\varphi\|_{\in \mathcal{C}^{1,1}}\right) .
$$

Proof The uniqueness is a consequence of the definition.

To prove the existence assume that $\rho$ is smooth. There are an increasing sequence $\varphi_{k}$ of smooth functions such that $\varphi_{k}$ tends to $\varphi$ in $\mathcal{C}^{1,1}$ norm. By Theorem 1 there is a solution $u_{k}$ of the following Dirichlet Problem

$$
\left\{\begin{array}{l}
u_{k} \in \mathcal{P} \mathcal{S H}(\Omega) \cap \mathcal{C}^{\infty}(\bar{\Omega}) \\
\left(i \partial \bar{\partial} u_{k}\right)^{n}=\frac{1}{k^{n}}(i \partial \bar{\partial} \rho)^{n} \text { in } \Omega \\
u_{k}=\varphi_{k} \text { on } \partial \Omega .
\end{array}\right.
$$

By Lemma $3.3\left\|u_{k}\right\|_{\mathcal{C}^{0,1}(\bar{\Omega})} \leq C\left(\|\rho\|_{\mathcal{C}^{0,1}(\Omega)}, m(\rho),\|\varphi\|_{\in \mathcal{C}^{1,1}}\right)$. Now we can put

$$
u:=\lim _{k \rightarrow \infty} u_{k} .
$$

It is enough to show that $u$ is a maximal function. Let a function $v \in \mathcal{P S H}(\Omega)$ be smaller than $u$ outside a compact subset of $\Omega$. From the comparison principle (Proposition 2.2) we obtain

$$
v+\frac{\rho}{k}-\sup _{\partial \Omega}\left(\varphi-\varphi_{k}\right) \leq u_{p}
$$

for $p \geq k$. Taking the limit we conclude that $v \leq u$ in $\Omega$. 
In the general case we can assume that $\varphi$ is a plurisubharmonic function on $\Omega$ (by adding $A \rho$ for $A$ enough large). We can approximate $\Omega$ by an increasing sequence of smooth strictly pseudoconvex domains $\Omega_{k}$ such that $\bigcup_{k} \Omega_{k}=\Omega$ and $\|\rho\|_{\mathcal{C}^{0,1}(\Omega)}, m(\rho)$ are under control, where $\rho_{k}$ are strictly plurisubharmonic smooth defining functions for $\Omega_{k}$. Let $u_{k}$ be a solution of the following Dirichlet Problem

$$
\left\{\begin{array}{l}
u_{k} \in \mathcal{P} \mathcal{S H}\left(\Omega_{k}\right) \cap \mathcal{C}\left(\bar{\Omega}_{k}\right) \\
u_{k} \text { is maximal } \\
u_{k}=\varphi \text { on } \partial \Omega_{k} .
\end{array}\right.
$$

Then $u_{k} \geq \varphi$, hence it is an increasing sequence and again we can put

$$
u:=\lim _{k \rightarrow \infty} u_{k} .
$$

If $v$ is as above, for every $\varepsilon>0$ we have $v-\varepsilon \leq u_{k}$ outside a compact set for $k$ large enough. So we obtain $v \leq u$ and conclude that $u$ is a maximal function as in the statement.

Note that in the above proposition it is not enough to assume that $\varphi$ is $\mathcal{C}^{1, \alpha}$ regular for some $\alpha<1$. Indeed, one can show that if $\Omega$ is strictly pseudoconvex, $P \in \partial \Omega$ and $\varphi(z) \leq \varphi(P)-(\operatorname{dist}(z, p))^{1+\alpha}$, then a solution of (4.1) is not Hölder continuous with the exponent greater than $\frac{1+\alpha}{2}$.

Theorem 4.2 (Harvey, Lawson [4]) There is a unique solution $u$ of the problem (4.1).

Proof Let $\varphi_{k}$ be an increasing sequence of smooth functions on $\bar{\Omega}$ such that $\lim _{k \rightarrow \infty} \varphi_{k}=\varphi$. By Proposition 4.1 there is a sequence $u_{k}$ of solutions of (4.1) with boundary conditions $\varphi_{k}$ (instead of $\varphi$ ). Because

$$
u_{k} \leq u_{p} \leq u_{k}+\sup _{\partial \Omega}\left(\varphi-\varphi_{k}\right)
$$

for $p \geq k$, the sequence $u_{k}$ is a Cauchy sequence in $\mathcal{C}(\bar{\Omega})$. Similar as in the previous proof we can conclude that its limit $u$ is a solution of the problem (4.1).

Note that we can also prove the above theorem directly from Theorem 1 .

The following proposition shows that being a continuous maximal plurisubharmonic function is a local property.

Proposition 4.3 Let $\Omega \subset M$ and $u \in \mathcal{P S H}(\Omega)$. Then

(i) If $u$ is maximal then $\left.u\right|_{U}$ is maximal for every $U \subset \Omega$;

(ii) If $\Omega$ is such that there is a bounded strictly plurisubharmonic function $\rho \in \mathcal{C}^{2}(\Omega)$, u is continuous and every point in $\Omega$ has a neighbourhood $U$ such that $\left.u\right|_{U}$ is maximal, then $u$ is maximal.

Proof (i) Suppose that $v \in \mathcal{P S H}(U)$ is such that $v \leq u$ outside a compact subset of $U$. Then $\max \{u, v\} \in \mathcal{P} \mathcal{S H}(\Omega)$ and we obtain $v \leq \max \{u, v\} \leq u$ on $U$.

(ii) We can assume that $\rho<0$. Let $\varepsilon>0, v \in \mathcal{P S H}(\Omega)$ and let $z_{0} \in \Omega$ be a point where a function $v+\varepsilon \rho-u$ attains its maximum. By (i) there is a strictly pseudoconvex domain $\tilde{\Omega} \subset \Omega$ with a smooth plurisubharmonic defining function $\tilde{\rho}$ such that $z_{0} \in \tilde{\Omega}$ and $\left.u\right|_{\tilde{\Omega}}$ is maximal. Note that there is $\tilde{\varepsilon}>0$ such that a function $\rho-\tilde{\varepsilon} \tilde{\rho}$ is plurisubharmonic in some neighbourhood of $\operatorname{cl}(\tilde{\Omega})$. Hence a function $\tilde{v}=\max \{v+\varepsilon \rho, v+\varepsilon(\rho-\tilde{\rho} \tilde{\varepsilon})\}$ is also plurisubharmonic and $\tilde{v}-u$ attains a maximum only in some compact subset of $\tilde{\Omega}$, which is impossible because $\left.u\right|_{\tilde{\Omega}}$ is maximal. As $\varepsilon$ and $v$ were arbitrary we can conclude that the function $u$ is maximal. 
In [4] the authors consider problem (4.1) for $\mathcal{F}(\mathcal{J})$-harmonic functions which they define in a different way than we define maximal functions but we will see that these concepts agree.

Let $\Omega \subset M$ and $u \in \mathcal{C} \cap \mathcal{P S H}(\Omega)$. We say that $u$ is $\mathcal{F}(\mathcal{J})$-harmonic if for every $U \subset \Omega$ and for every smooth strictly plurisubharmonic function $\phi \leq u$ on $U$ we have $\phi<u$ on $U$. One can show (using the comparison principle) that $\mathcal{C}^{2} \mathcal{F}(\mathcal{J})$-harmonic functions are exactly $\mathcal{C}^{2}$ solutions of (1) with $f=0$.

\section{Proposition 4.4 Let $\Omega$ and $u$ be as above. Then}

(i) If $u$ is maximal then $u$ is $\mathcal{F}(\mathcal{J})$-harmonic;

(ii) If $\Omega$ is such that there is a bounded strictly plurisubharmonic function $\rho \in \mathcal{C}^{2}(\Omega)$ and $u$ is $\mathcal{F}(\mathcal{J})$-harmonic, then $u$ is maximal.

Proof The first assertion follows from definitions. To proof (ii) we can assume, by Proposition 4.3, that $\Omega$ is a smooth strictly pseudoconvex domain with defining function $\rho$ such that $u \in \mathcal{C}(\bar{\Omega})$. Let $\varepsilon>0$. By Theorem 4.2 there is a continuous maximal plurisubharmonic function $u_{0}$ equal to $u$ on $\partial \Omega$. By Theorem 1 there is a smooth strictly plurisubharmonic function $u_{1}$ such that $u-\varepsilon<u_{1}<u$ on a boundary and

$$
\left(i \partial \bar{\partial} u_{1}\right)^{n}=\frac{1}{2} \varepsilon^{n}(i \partial \bar{\partial} \rho)^{n} .
$$

Then using the comparison principle (Proposition 2.2) we obtain

$$
u_{0}+\varepsilon \rho-\varepsilon \leq u_{1} \leq u \leq u_{0}
$$

and thus we get $u=u_{0}$.

Acknowledgments The author would like to express his gratitude to Z. Błocki for helpful discussions and advice during the work on this paper.

Open Access This article is distributed under the terms of the Creative Commons Attribution License which permits any use, distribution, and reproduction in any medium, provided the original author(s) and the source are credited.

\section{References}

1. Bertrand, F.: Local Analysis on Almost Complex Manifolds, PhD thesis (available on http://homepage. univie.ac.at/florian.bertrand/these.pdf) (2007)

2. Bertrand, F., Gaussier, H.: On the Gromov Hyperbolicity of Strongly Pseudoconvex Domains in Almost Complex Manifolds, arXiv:1202.4985

3. Caffarelli, L., Kohn, J.J., Nirenberg, L., Spruck, J.: The Dirichlet problem for non-linear second order elliptic equations II: complex Monge-Ampère, and uniformly elliptic equations. Commun. Pure Appl. Math. 38, 209-252 (1985)

4. Harvey, R., Lawson, B.: Potential Theory on Almost Complex Manifolds, ArXiv:1107.2584

5. Ivashkovich, S., Rosay, J.-P.: Schwarz-type lemmas for solutions of $\bar{\partial}$-inequalities and complete hyperbolicity of almost complex manifolds. Ann. Inst. Fourier (Grenoble) 54(7), 2387-2435 (2004)

6. McDuff, D.: Symplectic manifolds with contact type boundaries. Invent. Math. 103, 651-671 (1991)

7. Pali, N.: Fonctions plurisousharmoniques et courants positifs de type $(1,1)$ sur une variété presque complexe. Manuscripta Math. 118(3), 311-337 (2005)

8. Rosay, J.-P.: Notes on the Diederich-Sukhov-Tumanov normalization for almost complex structures. Collect. Math. 60(1), 43-62 (2009) 